\title{
Performance analysis of analog network coding based two-way amplify-and-forward system in mixed Rician and Nakagami-m fading environment
}

\author{
Muhammad Hasanain Chaudary* and Nandana Rajatheva
}

\begin{abstract}
This paper presents the performance analysis of analog network coding-based two-way amplify-and-forward relaying under mixed Rician and Nakagami-m fading environment. Closed form expressions for both the cumulative distribution function and probability distribution function of the instantaneous end-to-end SNR are derived. Using those, the closed form expressions for the first moment, second moment, and the symbol error rate (SER) for M-PSK modulated signals are obtained. The performance of the system is analyzed in terms of outage probability, average SER, and ergodic capacity. In addition, we investigate the outage probability for high SNR scenario to identify more details of the system performance in depth. Simulations are performed to verify the correctness of our theoretical analysis.
\end{abstract}

Keywords: Analog network coding (ANC), Amplify-and-forward (AF), Cumulative distribution function (CDF), Probability distribution function (PDF), Outage probability, Average symbol error rate (SER), Ergodic capacity

\section{Introduction}

Network coding (NC) was first introduced a decade ago [1], owing to its potential for improving the performance of a wireless network. In a wireless relay network, the application of network coding at the physical layer has been shown to increase the network throughput [2]. This network throughput is achieved by reducing the number of time slots required to exchange information between two source nodes $S_{1}$ and $S_{2}$ via a relay node $R$, from four to two. Four time slots have been used traditionally.

We can divide the prior related research into two main categories. The first category deals with the performance analysis over the symmetric fading channels for the relay networks (two-way, dual-hop, multi-hop, and multi-cast) [3-7]. The second category deals with the performance of relay networks over asymmetric fading channels $[8,9]$.

In the past years, the performance of relay networks (two-way, dual-hop, multi-hop, and multi-cast) over symmetric fading channels is being carried out by many

*Correspondence: chaudaryh@yahoo.com

Telecommunications, School of Engineering \& Technology, Asian Institute of Technology, Pathumthani, 12120 Thailand researchers. Work in [3] presents the outage probability and average symbol error rate (SER) analysis for twoway amplify-and-forward relaying over the symmetric (Nakagami- $m$ ) fading channel and in $[4,5]$ authors consider the symmetric (Rayleigh) fading channel. Along with network coding (NC) for two-way relaying the performance is studied in terms of outage probabaility and ergodic sum-rate in [6] over symmetric (Nakagami- $m$ ) fading channel, and in [7] the optimal transmission scheme analysis is carried out by computing the outage probability over symmetric (Rayleigh) fading channel.

In a relay network two sources are communicating with each other by using a single relay. Considering a practical scenario, relay network may have a line-ofsight (LOS) communication in one of the sides (source to relay link) and on the other side it may have only multipath communication (relay to destination link), and vice versa [8]. In such scenario using asymmetric fading channel is a promising solution. Recently, there is an increasing research interest on the performance of relay networks over asymmetric fading channels, and some recent examples of that are [8,9]. For the dual-hop

\section{Springer}

(c) 2012 Chaudary and Rajatheva; licensee Springer. This is an Open Access article distributed under the terms of the Creative Commons Attribution License (http://creativecommons.org/licenses/by/2.0), which permits unrestricted use, distribution, and reproduction in any medium, provided the original work is properly cited. 
amplify-and-forward relay transmission system, the performance in terms of deriving the closed form analytical expressions for cumulative distribution function (CDF) and probability density function (PDF) of end-end signalto-noise-ratio (SNR) over asymmetric (Nakagami-m and Rician) fading channels was assumed in [8]. The asymmetric (Rayleigh and Rician) fading channels have been investigated in [9] by deriving the exact and lower bound expressions for the outage probability and average bit error probability. None of these works have focused on the ergodic capacity analysis.

To the best of our knowledge the performance analysis over mixed Rician and Nakagami- $m$ asymmetric fading channels with analog network coding (ANC)-based two-way amplify-and-forward relay system has not been investigated before. Hence, in this paper we have adopted ANC-based two-way amplify-and-forward system in a mixed Rician and Nakagami- $m$ asymmetric fading environment. We have assumed source nodes $S_{1}$ and $S_{2}$ to be a base station (BS) and a mobile station (MS), respectively. We have used Rician fading channel between BS and relay node $R$ as they are assumed to have LOS communication and we have used Nakagami- $m$ fading channel between MS and relay node $R$ as they are considered to have only multipath communication. This particular setup is shown in Figure 1.

We will analyze the performance by evaluating the closed form expressions for outage probability, average SER (using the derived CDF), and ergodic capacity (using the derived PDF). We have plotted the analytical results using these derived close form expressions. Additionally, we investigate the outage probability for high SNR regime for a more comprehensive analysis of the system performance. At high SNR, the asymptotic outage probability that is obtained is very close to the exact outage probability. Remainder of the paper is organized as follows. Section 2 presents the system model. Performance and capacity analysis are discussed in Sections 3 and 4, respectively. Section 5 provides the asymptotic analysis. Results are given in Section 6, and Section 7 concludes the paper.

\section{System Model}

We consider a network coding-based two-way relaying system model in mixed Rician and Nakagami- $m$ fading environment as shown in Figure 1. Two source nodes $S_{1}$ and $S_{2}$ communicate through relay node $R$. The Rician fading (line-of-sight) environment is employed between the $S_{1} \rightarrow R$ link, where as Nakagami- $m$ fading environment is employed between the $S_{2} \rightarrow R$ link. The communications between the nodes is accomplished in two time slots. During the first time slot, both source nodes $S_{1}$ and $S_{2}$ transmit simultaneously to relay node $R$. During the second time slot, relay node $R$ normalizes the received signal-based on its transmit power constraint and broadcasts the combined signal to both source nodes. The source node knows its own information, thus the self information is discarded and the information of other source node is retrieved. We assume equal transmit power $P_{s}$ at source nodes $S_{1}, S_{2}$ and relay node $R$ and equal variance $N_{0}$ for the additive white Gaussian noise (AWGN) at the three nodes. The instantaneous end-to-end SNR at source node $S_{1}$ and source node $S_{2}$ during the second time slot, $\gamma_{S_{1}}$ and $\gamma_{S_{2}}$, can be given by $[3,4,6]$

$$
\begin{aligned}
& \gamma_{S_{1}}=\frac{\gamma_{1} \gamma_{2}}{2 \gamma_{1}+\gamma_{2}} \\
& \gamma_{S_{2}}=\frac{\gamma_{1} \gamma_{2}}{\gamma_{1}+2 \gamma_{2}},
\end{aligned}
$$

where $\gamma_{1}=P_{s}\left|h_{1}\right|^{2} / N_{0}$ and $\gamma_{2}=P_{s}\left|h_{2}\right|^{2} / N_{0}$ are the instantaneous SNRs of $S_{1} \rightarrow R$ and $S_{2} \rightarrow R$ links, respectively. The average transmit SNR is $\gamma_{0}=P_{s} / N_{0}$ and $\Omega_{1}=$ $\mathbb{E}\left\{h_{1}^{2}\right\}, \Omega_{2}=\mathbb{E}\left\{h_{2}^{2}\right\}$ are the respective variances of $h_{1}$ and $h_{2}$, where $\mathbb{E}\{\cdot\}$ represents the statistical average operator. Since $h_{1}$ experiences Rician fading, the instantaneous SNR $\gamma_{1}$ is a noncentral- $\chi^{2}$ distribution with PDF given by

$$
f_{\gamma_{1}}(\gamma)=\frac{(K+1) e^{-K}}{\overline{\gamma_{1}}} e^{-\frac{(K+1) \gamma}{\gamma_{1}}} I_{0}\left(2 \sqrt{\frac{K(K+1) \gamma}{\overline{\gamma_{1}}}}\right),
$$

where $K$ is a Rician $K$-factor defined as the ratio of the powers of the line-of-sight components to the scattered components, $\overline{\gamma_{1}}=\Omega_{1} \gamma_{0}$ is the average SNR of the $S_{1} \rightarrow R$ link, and $I_{0}$ is the zeroth order modified Bessel function of the first kind. For $K=0$, Rayleigh fading is experienced and $K=\infty$ gives the AWGN (no fading) situation. Also

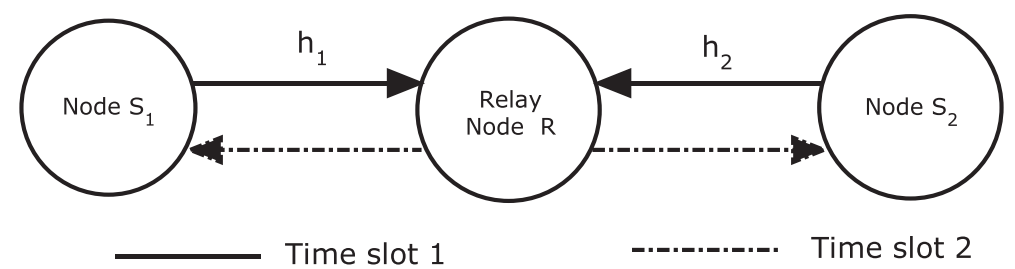

Figure 1 Block diagram of network coded-based two-way relay network. 
$h_{2}$ experiences Nakagami- $m$ fading, the PDF and the CDF of $\gamma_{2}$ are given by

$$
\begin{aligned}
& f_{\gamma_{2}}(\gamma)=\frac{m^{m}}{{\overline{\gamma_{2}}}^{m} \Gamma(m)} \gamma^{m-1} e^{\frac{m \gamma}{\gamma_{2}}} \\
& F_{\gamma_{2}}(\gamma)=1-\frac{\Gamma\left(m,\left(m / \overline{\gamma_{2}}\right) \gamma\right)}{\Gamma(m)},
\end{aligned}
$$

where $m$ is the Nakagami- $m$ fading parameter ranging from $\frac{1}{2}$ to $\infty, \Gamma(\cdot)$ is the gamma function [10, Equation 8.310.1], $\Gamma(\cdot, \cdot)$ is the incomplete gamma function [10, Equation 8.350.2] and $\overline{\gamma_{2}}=\Omega_{2} \gamma_{0}$ is the average SNR of the $R \rightarrow S_{2}$ link. For $m=\frac{1}{2}$, it exibits one-sided Gaussian distribution, for $m=1$, it reduces to Rayleigh distribution and it converges to a nonfading AWGN channel as $m \rightarrow \infty$.

\section{Performance analysis Outage probability}

The data is assumed to be correctly received at the source node $S_{1}$ when the equivalent receives SNR is greater than or equal to threshold SNR $\gamma_{\mathrm{t} h}$. When the received SNR at the source node $S_{1}$ is below the threshold SNR $\gamma_{\text {th }}$ the receiver declares an outage. Thus the outage probability at the source node $S_{1}$ can be expressed as

$$
P_{\text {out }, S_{1}}\left(\gamma_{\mathrm{th}}\right)=\operatorname{Pr}\left(\gamma_{S_{1}}<\gamma_{\mathrm{t} h}\right)=F_{\gamma_{s_{1}}}\left(\gamma_{\mathrm{th}}\right),
$$

where $F_{\gamma_{s_{1}}}\left(\gamma_{\mathrm{t} h}\right)$ is the CDF of the instantaneous SNR at source node $S_{1}$, evaluated at $\gamma=\gamma_{\text {th }}$ as

$$
\begin{aligned}
& F_{\gamma_{S_{1}}}\left(\gamma_{\mathrm{t} h}\right)=\int_{0}^{\infty} \operatorname{Pr}\left(\frac{\gamma_{1} \gamma_{2}}{2 \gamma_{1}+\gamma_{2}} \leq \gamma_{\mathrm{t} h} \mid \gamma_{1}\right) f_{\gamma_{1}}\left(\gamma_{1}\right) \mathrm{d} \gamma_{1} \\
& =\int_{0}^{\gamma_{\text {th }}} \operatorname{Pr}\left(\gamma_{2} \geq \frac{2 \gamma_{\mathrm{th}} \gamma_{1}}{\gamma_{1}-\gamma_{\mathrm{th}}} \mid \gamma_{1}\right) f_{\gamma_{1}}\left(\gamma_{1}\right) \mathrm{d} \gamma_{1} \\
& +\int_{\gamma_{\text {th }}}^{\infty} \operatorname{Pr}\left(\gamma_{2} \leq \frac{2 \gamma_{\text {th }} \gamma_{1}}{\gamma_{1}-\gamma_{\text {th }}} \mid \gamma_{1}\right) f_{\gamma_{1}}\left(\gamma_{1}\right) \mathrm{d} \gamma_{1} \\
& =\int_{0}^{\gamma_{\text {th }}} f_{\gamma_{1}}\left(\gamma_{1}\right) \mathrm{d} \gamma_{1}+\int_{\gamma_{\text {th }}}^{\infty} F_{\gamma_{2}}\left(\frac{2 \gamma_{\mathrm{th}} \gamma_{1}}{\gamma_{1}-\gamma_{\mathrm{th}}}\right) f_{\gamma_{1}}\left(\gamma_{1}\right) \mathrm{d} \gamma_{1} \\
& =\frac{(K+1) e^{-K}}{\bar{\gamma}_{1}} \int_{0}^{\gamma_{\text {th }}} e^{-\frac{(K+1) \gamma_{1}}{\gamma_{1}}} I_{0}\left(2 \sqrt{\frac{K(K+1) \gamma_{\text {th }}}{\gamma_{1}}}\right) \mathrm{d} \gamma_{1} \\
& +\frac{(K+1) e^{-K}}{\overline{\gamma_{1}}} \int_{\gamma_{\mathrm{th}}}^{\infty} e^{-\frac{(K+1) \gamma_{1}}{\bar{\gamma}_{1}}} I_{0}\left(2 \sqrt{\frac{K(K+1) \gamma_{\mathrm{th}}}{\bar{\gamma}_{1}}}\right) \mathrm{d} \gamma_{1} \\
& -\frac{(K+1) e^{-K}}{\overline{\gamma_{1}} \Gamma(m)} \int_{\gamma_{\mathrm{th}}}^{\infty} e^{-\frac{(K+1) \gamma_{1}}{\bar{\gamma}_{1}}} \Gamma\left(m, \frac{2 \gamma_{1}}{\gamma_{1}-\gamma_{\mathrm{th}}} \frac{m \gamma_{\mathrm{th}}}{\bar{\gamma}_{2}}\right) \\
& \times I_{0}\left(2 \sqrt{\frac{K(K+1) \gamma_{\text {th }}}{\overline{\gamma_{1}}}}\right) \mathrm{d} \gamma_{1}
\end{aligned}
$$

After simplifying (7) using the identities [10, Equations 8.447.1, 3.351.1, 3.351.2, 8.352.1, and 8.352.2], we get

$$
\begin{aligned}
F_{\gamma_{s_{1}}}\left(\gamma_{\mathrm{th}}\right)= & e^{-K} \sum_{g=0}^{\infty} \frac{K^{g}}{g !}-\frac{(K+1) e^{-K}}{\overline{\gamma_{1}} \Gamma(m)} \sum_{g=0}^{\infty} \frac{K^{g}(K+1)^{g}}{(g !)^{2} \bar{\gamma}_{1}^{g}} \\
& \int_{\gamma_{\mathrm{th}}}^{\infty} \gamma_{1}^{g} e^{-\frac{(K+1)}{\overline{\gamma_{1}}} \gamma_{1}} \Gamma\left(m, \frac{2 \gamma_{1}}{\gamma_{1}-\gamma_{\mathrm{th}}} \frac{m \gamma_{\mathrm{th}}}{\bar{\gamma}_{2}}\right) \mathrm{d} \gamma_{1}
\end{aligned}
$$

After applying the identity $e^{K}=\sum_{g=0}^{\infty} \frac{K^{g}}{g !}$ given in [10, Equation 1.211.1], the term $e^{-K} \sum_{g=0}^{\infty} \frac{K^{g}}{g !}$ in (8) reduces to $1\left(e^{-K} \sum_{g=0}^{\infty} \frac{K^{g}}{g !}=e^{-K} e^{K}=1\right)$, and also the integral term can be expressed in its general form as

$$
\begin{aligned}
F_{\gamma_{s_{1}}}\left(\gamma_{\mathrm{t} h}\right)= & 1-\frac{(K+1) e^{-K}}{\overline{\gamma_{1} \Gamma(m)}} \sum_{g=0}^{\infty} \frac{K^{g}(K+1)^{g}}{(g !)^{2} \bar{\gamma}^{g}} \\
& \times \int_{y}^{\infty} x^{g} e^{-a x} \Gamma\left(c, 2 b+\frac{2 b}{x-y}\right) \mathrm{d} x
\end{aligned}
$$

where $a=(K+1) / \overline{\gamma_{1}}, b=m_{2} \gamma_{\mathrm{th}} / \overline{\gamma_{2}}, c=m_{2}, x=\gamma_{1}$, and $y=\gamma_{\mathrm{t} h}$. The incomplete gamma function of the integral in (9) can be written in the following form using identities [10, Equations 8.352.2, and 1.111]

$$
\begin{aligned}
\Gamma\left(c, 2 b+\frac{2 b}{x-y}\right)= & (c-1) ! e^{-\left(2 b+\frac{2 b y}{x-y}\right)} \sum_{q=0}^{c-1} \sum_{v=0}^{q} \frac{(2 b)^{q}}{q !} \\
& \times\left(\begin{array}{c}
q \\
v
\end{array}\right)\left(\frac{y}{x-y}\right)^{v}
\end{aligned}
$$

Using (10) in (9), we get

$$
\begin{aligned}
F_{\gamma_{s_{1}}}\left(\gamma_{\mathrm{t} h}\right)= & 1-\frac{(K+1) e^{-K}}{\overline{\gamma_{1} \Gamma(m)}} \sum_{g=0}^{\infty} \frac{K^{g}(K+1)^{g}}{(g !)^{2} \bar{\gamma}^{g}}(c-1) ! e^{-2 b} \\
& \sum_{q=0}^{c-1} \sum_{v=0}^{q} \frac{(2 b)^{q}}{q !}\left(\begin{array}{c}
q \\
v
\end{array}\right) \int_{y}^{\infty} x^{g} e^{\left(-a x-\frac{2 b y}{x-y}\right)}\left(\frac{y}{x-y}\right)^{v} \mathrm{~d} x
\end{aligned}
$$

The inner integral in (11) can be evaluated with the help of [10, Equation 3.471.9] yielding the compact closedform CDF expression

$$
\begin{aligned}
F_{\gamma_{s_{1}}}\left(\gamma_{\mathrm{t} h}\right)= & 1-\frac{2(K+1)(m-1) ! \exp \left(-K-\left(\frac{2 m}{\overline{\overline{2}_{2}}}+\frac{K+1}{\bar{\gamma}_{1}}\right) \gamma_{\mathrm{th}}\right)}{\overline{\gamma_{1}} \Gamma(m)} \\
& \times \sum_{g=0}^{\infty} \sum_{q=0}^{m-1} \sum_{v=0}^{q} \sum_{l=0}^{g} \frac{1}{(g !)^{2} q !}\left(\begin{array}{c}
q \\
v
\end{array}\right)\left(\begin{array}{l}
g \\
l
\end{array}\right)\left(\frac{K(K+1)}{\overline{\gamma_{1}}}\right)^{g} \\
& \times\left(\frac{2 m \gamma_{\mathrm{th}}}{\overline{\gamma_{2}}}\right)^{q+\frac{l-v+1}{2}}\left(\frac{\overline{\gamma_{1}}}{K+1}\right)^{\frac{l-v+1}{2}} \\
& \times \gamma_{\mathrm{th}}^{g+\frac{v-l+1}{2}} \mathbf{K}_{l-v+1}\left(2 \gamma_{\mathrm{th}} \sqrt{\frac{2 m(K+1)}{\overline{\gamma_{2} \gamma_{1}}}}\right)
\end{aligned}
$$

where $\mathbf{K}_{u}($.$) is the u$-th order modified Bessel function of the second kind defined in [10, Equation 8.432.6]. Using the closed form CDF expression (12) in (6), the analytical probability of outage for two-way AF relaying network over asymmetric (Rician and Nakagami- $m$ ) fading 


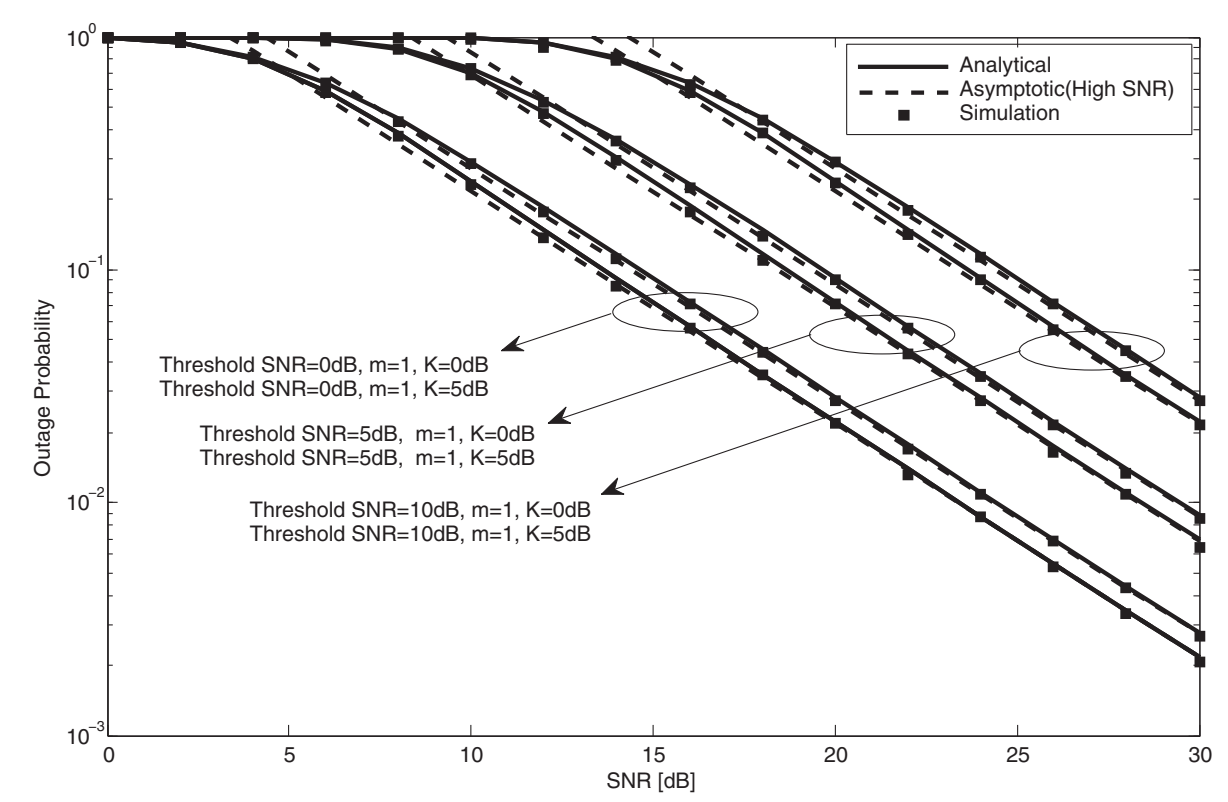

Figure 2 Outage probability of ANC-based two-way relay system with $m=1$.

channels can be easily expressed in the closed form. The analytical outage probability versus SNR curves are plotted in Figures 2, 3 and 4 for different values of threshold $\operatorname{SNR}\left(\gamma_{\mathrm{t} h}\right), K$, and $m$. As can be seen from (12), the fading parameter $m$ and $K$ affects the outage probability. When $m=1$, Rayleigh fading is experienced as a special case. In Section 5, we compared both the analytical and simulation results of the outage probability with the derived asymptotic outage probability expression to better understand the system performance at high SNR regions.

\section{Average symbol error rate (SER)}

In this subsection we derive the closed form expression of average SER. For a wide range of modulation schemes the formula for average SER as given in [11]

$$
\mathrm{SER}=a E_{\gamma}[Q(\sqrt{2 b \gamma})]
$$

where $Q(x)=\frac{1}{\sqrt{2 \pi}} \int_{x}^{\infty} e^{-\frac{y^{2}}{2}} \mathrm{~d} y$ and $[a, b>0]$, which defines the parameters for the modulation schemes. For

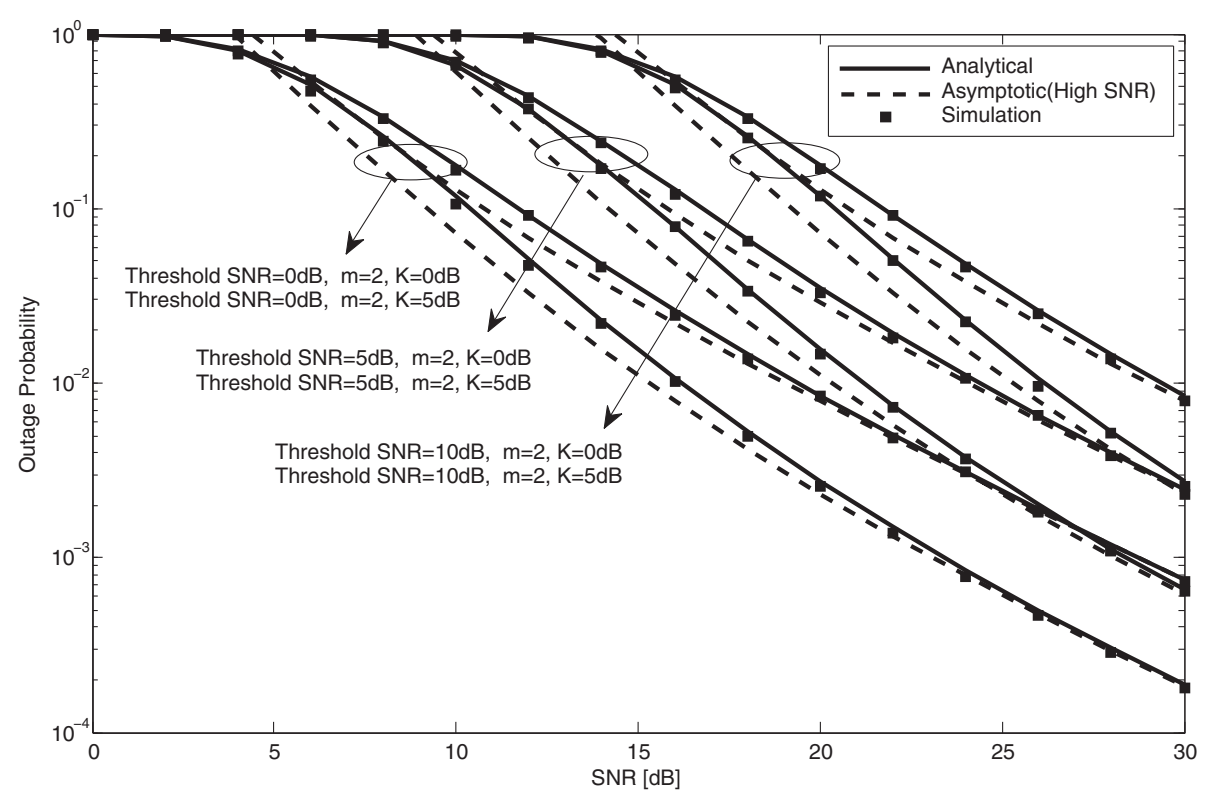

Figure 3 Outage probability of ANC-based two-way relay system with $m=2$. 


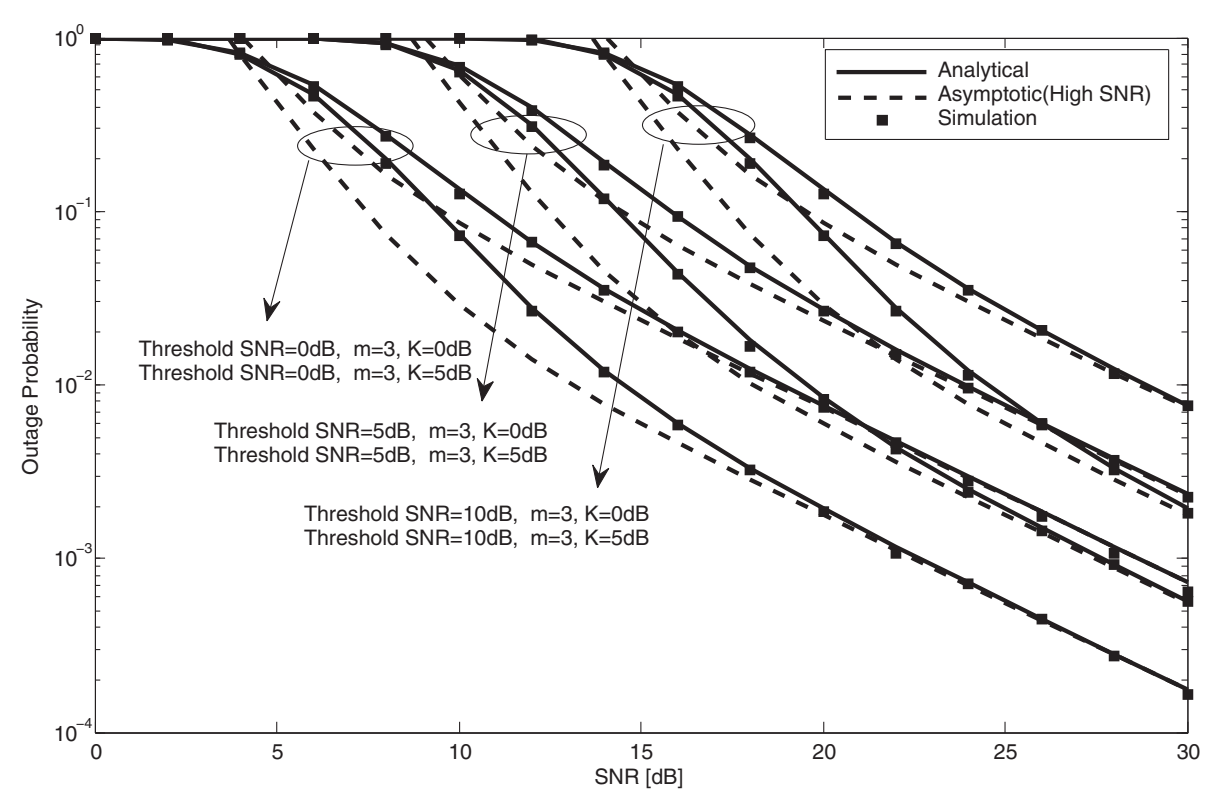

Figure 4 Outage probability of ANC-based two-way relay system with $m=3$.

example for BPSK $a=1$ and $b=1$, and for QPSK $a=1$ and $b=0.5$. Integration by parts of (13) leads to

$$
\mathrm{SER}=\frac{a \sqrt{b}}{2 \sqrt{\pi}} \int_{0}^{\infty} \frac{F_{\gamma_{1}}(z)}{\sqrt{z}} e^{-b z} \mathrm{~d} z
$$

Substituting (12) in (14), we get

$$
\begin{aligned}
\mathrm{SER}= & \frac{a \sqrt{b}}{2 \sqrt{\pi}} \int_{0}^{\infty} \frac{e^{-b \gamma_{\mathrm{t} h}}}{\sqrt{\gamma_{\mathrm{t} h}}} \mathrm{~d} \gamma_{\mathrm{t} h}-\frac{a \sqrt{b}}{\sqrt{\pi}} \frac{(K+1)(m-1) ! e^{-K}}{\overline{\gamma_{1} \Gamma(m)}} \\
& \sum_{g=0}^{\infty} \sum_{q=0}^{m-1} \sum_{\nu=0}^{q} \sum_{l=0}^{g} \frac{1}{(g !)^{2} q !}\left(\begin{array}{c}
q \\
v
\end{array}\right)\left(\begin{array}{c}
g \\
l
\end{array}\right)\left(\frac{K(K+1)}{\overline{\gamma_{1}}}\right)^{g}\left(\frac{\overline{\gamma_{1}}}{K+1}\right)^{\frac{l-v+1}{2}} \\
& \left(\frac{2 m}{\overline{\gamma_{2}}}\right)^{q+\frac{l-v+1}{2}} \int_{0}^{\infty} \gamma_{\mathrm{t} h}^{q+g+\frac{3}{2}-1} \exp \left(-\left(b+\frac{2 m}{\overline{\gamma_{2}}}+\frac{K+1}{\overline{\gamma_{1}}}\right) \gamma_{\mathrm{t} h}\right) \\
& \mathbf{K}_{l-v+1}\left(2 \gamma_{\mathrm{th}} \sqrt{\frac{2 m(K+1)}{\overline{\gamma_{2} \gamma_{1}}}}\right) \mathrm{d} \gamma_{\mathrm{t} h}
\end{aligned}
$$

The inner integrals in (15) can be evaluated using the identities [10, Equations 3.361.2 and 6.621.3] to yield the closed-form SER expression

$$
\begin{aligned}
& \mathrm{SER}=\frac{a}{2}-\frac{a \sqrt{b} e^{-K}(K+1)(m-1) !}{\overline{\gamma_{1}} \Gamma(m)} \sum_{g=0}^{\infty} \sum_{q=0}^{m-1} \sum_{v=0}^{q} \sum_{l=0}^{g} \frac{1}{(g !)^{2} q !} \\
& \left(\begin{array}{l}
q \\
v
\end{array}\right)\left(\begin{array}{l}
g \\
l
\end{array}\right)\left(\frac{K(K+1)}{\overline{\gamma_{1}}}\right)^{g}\left(\frac{2 m}{\overline{\gamma_{2}}}\right)^{q+\frac{l-v+1}{2}}\left(\frac{\overline{\gamma_{1}}}{K+1}\right)^{\frac{l-v+1}{2}} \\
& \frac{\left(4 \sqrt{\frac{2 m(K+1)}{\gamma_{2} \gamma_{1}}}\right)^{l-v+1}}{\left(b+\frac{2 m}{\gamma_{2}}+\frac{K+1}{\gamma_{1}}+2 \sqrt{\frac{2 m(K+1)}{\bar{\gamma}_{2} \gamma_{1}}}\right)^{q+g+l-v+\frac{5}{2}}} \frac{\Gamma\left(q+g+l-v+\frac{5}{2}\right) \Gamma\left(q+g-l+v+\frac{1}{2}\right)}{\Gamma(q+g+2)} \\
& \mathrm{F}\left(q+g+l-v+\frac{5}{2}, l-v+\frac{3}{2} ; q+g+2 ; \frac{b+\frac{2 m}{\gamma_{2}}+\frac{K+1}{\gamma_{1}}-2 \sqrt{\frac{2 m(K+1)}{\gamma_{2} \gamma_{1}}}}{b+\frac{2 m}{\gamma_{2}}+\frac{K+1}{\gamma_{1}}+2 \sqrt{\frac{2 m(K+1)}{\gamma_{2} \gamma_{1}}}}\right),
\end{aligned}
$$

where $\mathrm{F}(\cdot, \cdot ; \cdot ; \cdot)$ is the hypergeometric function defined in [10, Equation 9.111], $\Gamma(\cdot)$ is the gamma function defined in [10, Equation 8.310.1], $a$ and $b$ defines the parameters for the modulation schemes. The closed form average SER expression in (16) provides the analytical results over asymmetric (Rician and Nakagami-m) fading channels. For different values of $K$ and $m$, the analytical average SER versus SNR curves are plotted in Figures 5, 6 and 7 for $M$-ary modulation such as $M$-PSK (BPSK and QPSK). It can be seen from (16), that by fixing value of $m$ at the MS and changing the value of $K$ at the BS, we can achieve a significant performance. For instance, at $m=3$, we can observe the best case scenario.

\section{Capacity analysis \\ Ergodic capacity}

The ergodic capacity is defined in [12] as the expectation of the mutual information rate over the channel distribution between the source and the destination as

$$
C_{\mathrm{erg}}=E\left[\frac{1}{2} \log \left(1+\gamma_{S_{1}}\right)\right]=\log _{2} e . E\left[\frac{1}{2} \ln \left(1+\gamma_{S_{1}}\right)\right]
$$

We obtain the exact closed form of approximate ergodic capacity expression of ANC-based two-way AF relaying system in a mixed Rician and Nakagami- $m$ fading channels using the Taylor expansion of $\ln \left(1+\gamma_{S_{1}}\right)$, involving 


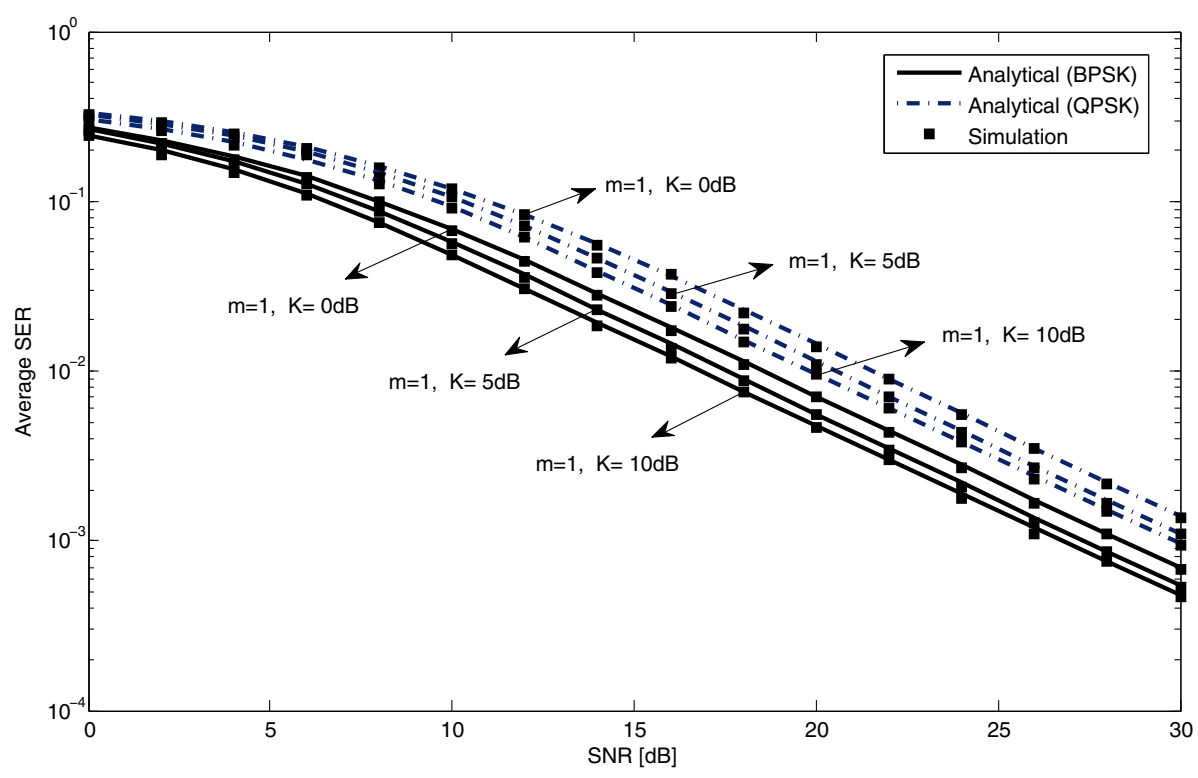

Figure 5 Average SER of ANC-based two-way relay system with $m=1$.

the expectation $E\left\{\gamma_{\mathrm{t} h}\right\}$ and the power $E\left\{\gamma_{\mathrm{th}}^{2}\right\}$ to obtain the second-order approximation for $C_{\text {erg }}$ given in [12] as

$$
C_{\mathrm{erg}} \approx \frac{1}{2} \log _{2} e .\left[\ln \left(1+E\left\{\gamma_{\mathrm{th}}\right\}\right)-\frac{E\left\{\gamma_{\mathrm{th}}^{2}\right\}-\left(E\left\{\gamma_{\mathrm{th}}\right\}\right)^{2}}{2\left(1+E\left\{\gamma_{\mathrm{th}}\right\}\right)^{2}}\right]
$$

In order to obtain the first moment (mean) $E\left\{\gamma_{\text {th }}\right\}$ and second moment $E\left\{\gamma_{\mathrm{th}}^{2}\right\}$, we need first to get the probability density function (PDF) of the instantaneous SNR at source node $S_{1}$. This PDF can be found by taking the first derivative of (12) with respect of $\gamma_{\mathrm{t} h}$, yielding

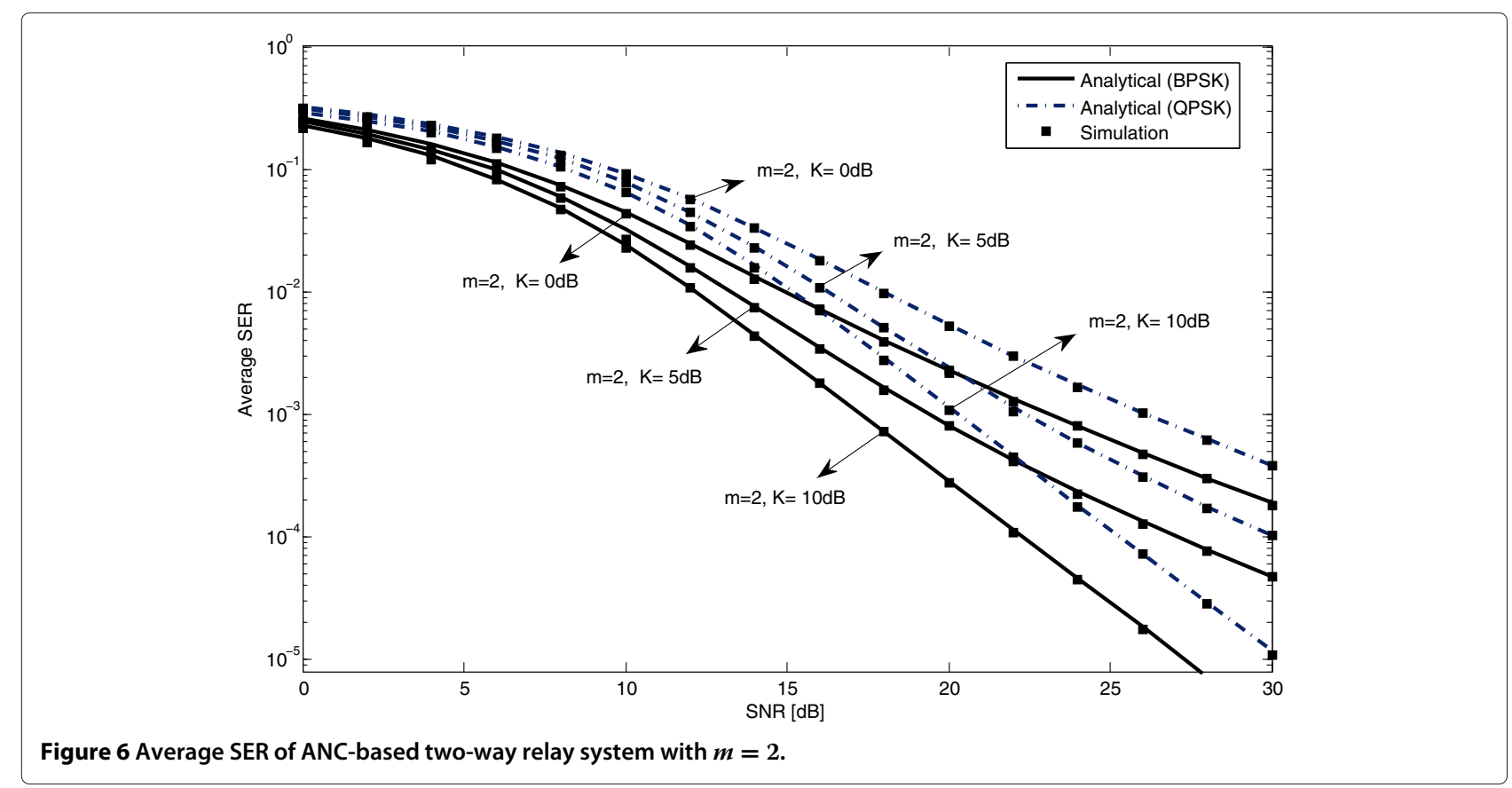

Figure 6 Average SER of ANC-based two-way relay system with $\boldsymbol{m}=\mathbf{2}$. 


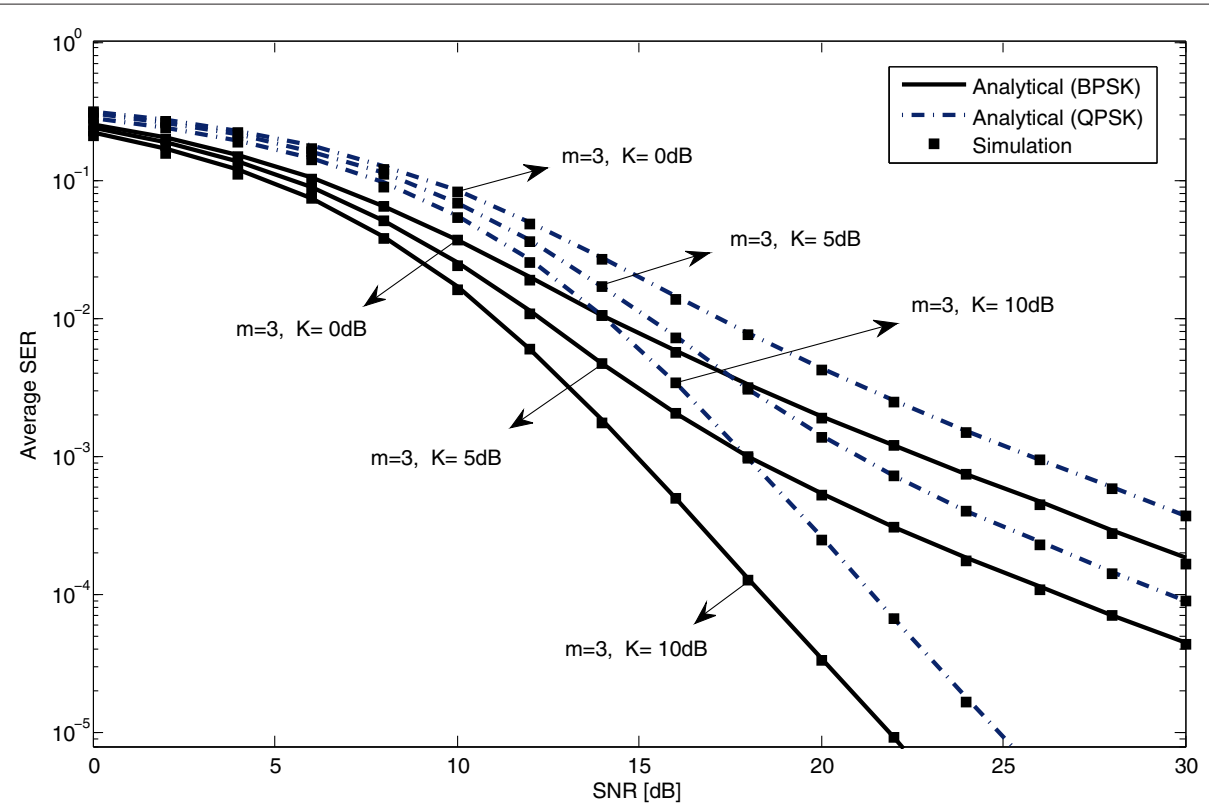

Figure 7 Average SER of ANC-based two-way relay system with $m=3$.

where $U_{1}\left(\gamma_{\mathrm{th}}\right)=e^{-\left(\frac{2 m}{\gamma_{2}}+\frac{K+1}{\gamma_{1}}\right) \gamma_{\mathrm{th}}}, \quad U_{2}\left(\gamma_{\mathrm{th}}\right)=x=\left[U_{1}^{\prime}\left(\gamma_{\mathrm{th}}\right) U_{2}\left(\gamma_{\mathrm{th}}\right)+U_{1}\left(\gamma_{\mathrm{th}}\right) U_{2}^{\prime}\left(\gamma_{\mathrm{th}}\right)\right]$

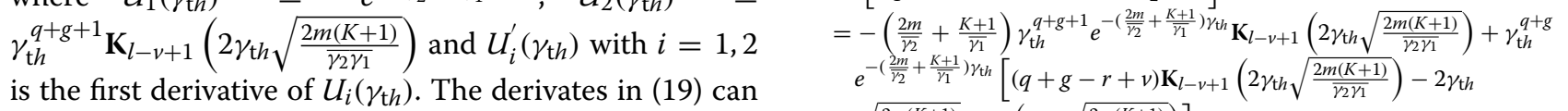
be evaluated with the help of [10, Equation 8.486.12], yielding

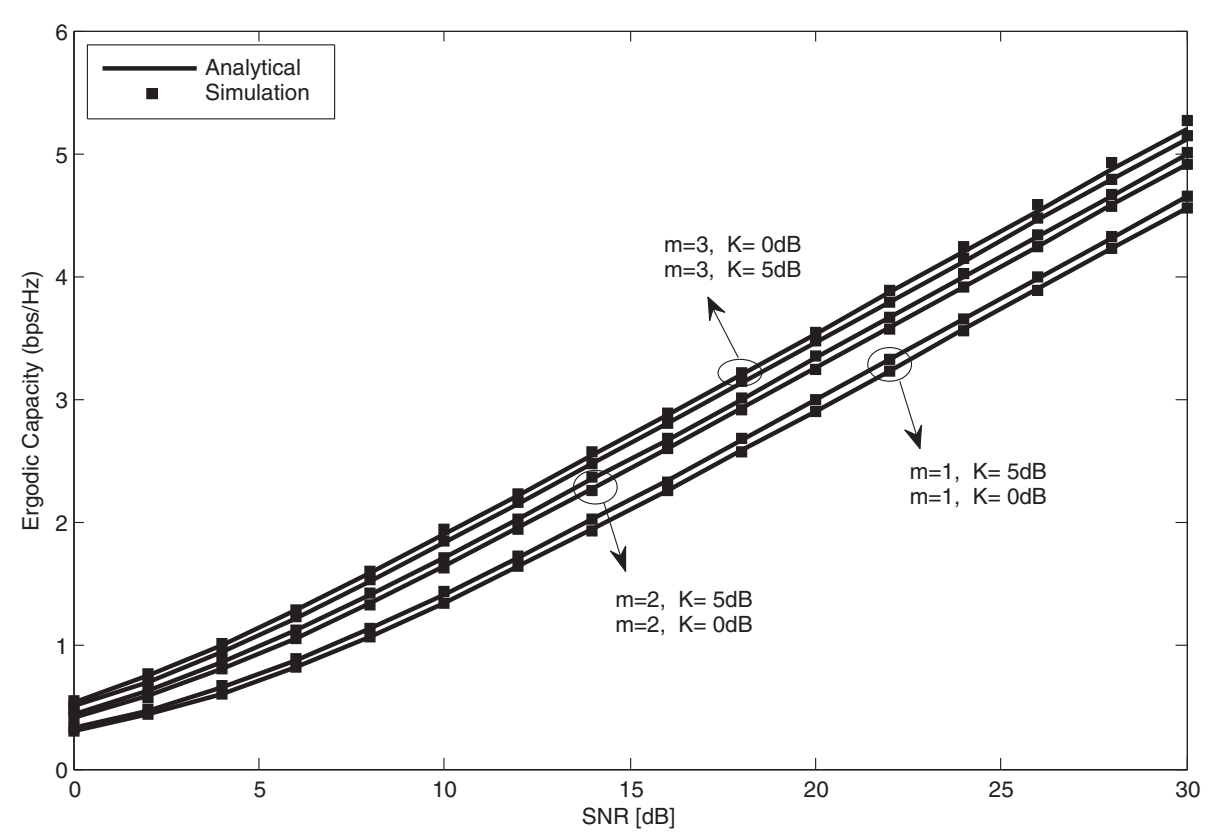

Figure 8 Ergodic capacity of ANC-based two-way relay system with different $m$ and $K$ values. 
After substituting (20) in (19), we get the closed form PDF expression as follows

$$
\begin{aligned}
f_{\gamma_{S_{1}}}\left(\gamma_{\mathrm{t} h}\right)= & \frac{2(K+1)(m-1) ! e^{-K}}{\bar{\gamma}_{1} \Gamma(m)} \sum_{g=0}^{\infty} \sum_{q=0}^{m-1} \sum_{v=0}^{q} \sum_{l=0}^{g} \frac{1}{(g !)^{2} q !}\left(\begin{array}{c}
q \\
v
\end{array}\right)\left(\begin{array}{l}
g \\
l
\end{array}\right) \\
& \left(\frac{K(K+1)}{\overline{\gamma_{1}}}\right)^{g}\left(\frac{2 m \gamma_{\mathrm{th}}}{\overline{\gamma_{2}}}\right)^{q+\frac{l-v+1}{2}}\left(\frac{\overline{\gamma_{1}}}{K+1}\right)^{\frac{l-v+1}{2}} \gamma_{\mathrm{th}}^{q+g} e^{-\left(\frac{2 m}{\overline{\gamma_{2}}}+\frac{K+1}{\bar{\gamma}_{1}}\right) \gamma_{\mathrm{th}}} \\
& {\left[\mathbf{K}_{l-v+1}\left(2 \gamma_{\mathrm{th}} \sqrt{\frac{2 m(K+1)}{\overline{\gamma_{2} \gamma_{1}}}}\right)\left(\gamma_{\mathrm{th}}\left(\frac{2 m}{\overline{\gamma_{2}}}+\frac{K+1}{\overline{\gamma_{1}}}\right)-(q+g-r+v)\right)\right.} \\
& \left.+2 \gamma_{\mathrm{th}} \sqrt{\frac{2 m(K+1)}{\overline{\gamma_{2} \gamma_{1}}}} \mathbf{K}_{l-v}\left(2 \gamma_{\mathrm{th}} \sqrt{\frac{2 m(K+1)}{\overline{\gamma_{2} \gamma_{1}}}}\right)\right]
\end{aligned}
$$

Thus, the first moment (mean) $E\left\{\gamma_{\mathrm{th}}\right\}$ and second moment $E\left\{\gamma_{\mathrm{t} h}^{2}\right\}$ can be obtained by using the closed form PDF expression in (21) and the identity [10, Equation 6.621.3] as

$$
\begin{aligned}
& E\left\{\gamma_{\mathrm{t} h}\right\}=\int_{0}^{\infty} \gamma_{\mathrm{th}} f_{\gamma_{S_{1}}}\left(\gamma_{\mathrm{th}}\right) \mathrm{d} \gamma_{\mathrm{t} h} \\
& =\frac{2 e^{-K}(K+1)(m-1) !}{\overline{\gamma_{1} \Gamma(m)}} \sum_{g=0}^{\infty} \sum_{q=0}^{m-1} \sum_{v=0}^{q} \sum_{l=0}^{g} \frac{1}{(g !)^{2} q !}\left(\begin{array}{c}
q \\
v
\end{array}\right)\left(\begin{array}{l}
g \\
l
\end{array}\right) \\
& \left(\frac{K(K+1)}{\overline{\gamma_{1}}}\right)^{g}\left(\frac{\overline{\gamma_{1}}}{K+1}\right)^{\frac{l-v+1}{2}}\left(\frac{2 m}{\overline{\gamma_{2}}}\right)^{q+\frac{l-v+1}{2}} \frac{\sqrt{\pi}\left(4 \sqrt{\frac{2 m(K+1)}{\bar{\gamma}_{2} \gamma_{1}}}\right)^{l-v+1}}{\left(\frac{2 m}{\overline{\gamma_{2}}}+\frac{K+1}{\overline{\gamma_{1}}} 2 \sqrt{\frac{2 m(K+1)}{\overline{\gamma_{2} \gamma_{1}}}}\right)^{q+g+l-v+3}} \\
& \left(\frac{\frac{2 m}{\gamma_{2}}+\frac{K+1}{\gamma_{1}}}{\frac{2 m}{\bar{\gamma}_{2}}+\frac{K+1}{\gamma_{1}}+2 \sqrt{\frac{2 m(K+1)}{\gamma_{2} \gamma_{1}}}} \frac{\Gamma(q+g+l-v+4) \Gamma(q+g-l+v+2)}{\Gamma\left(q+g+\frac{7}{2}\right)}\right. \\
& \mathrm{F}\left(q+g+l-v+4, l-v+\frac{3}{2} ; q+g+\frac{7}{2} ; \frac{\frac{2 m}{\bar{\gamma}_{2}}+\frac{K+1}{\gamma_{1}}-2 \sqrt{\frac{2 m(K+1)}{\overline{2}^{2} \gamma_{1}}}}{\frac{\bar{\gamma}_{2}}{\gamma_{2}}+\frac{K+1}{\gamma_{1}}+2 \sqrt{\frac{2 m(K+1)}{\bar{V}_{2} \gamma_{1}}}}\right) \\
& -(q+g-l+v) \frac{\Gamma(q+g+l-v+3) \Gamma(q+g-l+v+1)}{\Gamma\left(q+g+\frac{5}{2}\right)} \\
& \mathrm{F}\left(q+g+l-v+3, l-v+\frac{3}{2} ; q+g+\frac{5}{2} ; \frac{\frac{2 m}{\bar{\gamma}_{2}}+\frac{K+1}{\gamma_{1}}-2 \sqrt{\frac{2 m(K+1)}{\gamma_{2} \gamma_{1}}}}{\frac{K+1}{\bar{\gamma}_{2}}+2 \sqrt{\frac{2 m(K+1)}{\gamma_{2} \gamma_{1}}}}\right) \\
& +2 \frac{\Gamma(q+g+l-v+3) \Gamma(q+g-l+v+3)}{\Gamma\left(q+g+\frac{7}{2}\right)} \\
& \left.\mathrm{F}\left(q+g+l-v+3, l-v+\frac{1}{2} ; q+g+\frac{7}{2} ; \frac{\frac{2 m}{\overline{\gamma_{2}}}+\frac{K+1}{\overline{\gamma_{1}}}-2 \sqrt{\frac{2 m(K+1)}{\overline{\gamma_{j} \gamma_{1}}}}}{\frac{2 m}{\overline{\gamma_{2}}}+\frac{K+1}{\overline{\gamma_{1}}}+2 \sqrt{\frac{2 m(K+1)}{\bar{\gamma}_{2} \gamma_{1}}}}\right)\right)
\end{aligned}
$$

and

$$
\begin{aligned}
& E\left\{\gamma_{\mathrm{th}}^{2}\right\}=\int_{0}^{\infty} \gamma_{\mathrm{th}}^{2} f_{S_{\mathrm{l}}}\left(\gamma_{\mathrm{t} h}\right) \mathrm{d} \gamma_{\mathrm{th}} \text { of } \gamma_{\mathrm{th}} \\
& =\frac{2 e^{-K}(K+1)(m-1) !}{\overline{\gamma_{1} \Gamma(m)}} \sum_{g=0}^{\infty} \sum_{q=0}^{m-1} \sum_{v=0}^{q} \sum_{l=0}^{g} \frac{1}{(g !)^{2} q !}\left(\begin{array}{l}
q \\
v
\end{array}\right)\left(\begin{array}{l}
g \\
l
\end{array}\right) \\
& \left(\frac{K(K+1)}{\overline{\gamma_{1}}}\right)^{g}\left(\frac{\overline{\gamma_{1}}}{K+1}\right)^{\frac{l-v+1}{2}}\left(\frac{2 m}{\overline{\gamma_{2}}}\right)^{q+\frac{l-v+1}{2}} \frac{\sqrt{\pi}\left(4 \sqrt{\frac{2 m(K+1)}{\bar{\gamma}_{2} \gamma_{1}}}\right)^{l-v+1}}{\left(\frac{2 m}{\overline{\gamma_{2}}}+\frac{K+1}{\overline{\gamma_{1}}} 2 \sqrt{\frac{2 m(K+1)}{\overline{\gamma_{2} \gamma_{1}}}}\right)^{q+g+l-v+4}} \\
& \left(\frac{\frac{2 m}{\gamma_{2}}+\frac{K+1}{\gamma_{1}}}{\frac{2 m}{\bar{V}_{2}}+\frac{K+1}{\gamma_{1}}+2 \sqrt{\frac{2 m(K+1)}{\gamma_{2} \gamma_{1}}}} \frac{\Gamma(q+g+l-v+5) \Gamma(q+g-l+v+3)}{\Gamma\left(q+g+\frac{9}{2}\right)}\right. \\
& \mathrm{F}\left(q+g+l-v+5, l-v+\frac{3}{2} ; q+g+\frac{9}{2} ; \frac{\frac{2 m}{\bar{\gamma}_{2}}+\frac{K+1}{\bar{\gamma}_{1}}-2 \sqrt{\frac{2 m(K+1)}{\bar{\gamma}_{2} \gamma_{1}}}}{\frac{\bar{\gamma}_{2}}{\gamma_{2}}+\frac{K+1}{\gamma_{1}}+2 \sqrt{\frac{2 m(K+1)}{\bar{\gamma}_{2} \gamma_{1}}}}\right) \\
& -(q+g-l+v) \frac{\Gamma(q+g+l-v+4) \Gamma(q+g-l+v+2)}{\Gamma\left(q+g+\frac{7}{2}\right)}
\end{aligned}
$$

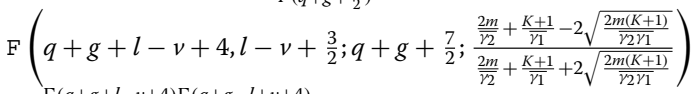

$$
\begin{aligned}
& +2 \frac{\Gamma(q+g+l-v+4) \Gamma(q+g-l+v+4)}{\Gamma\left(q+g+\frac{9}{2}\right)} \\
& \left.\mathrm{F}\left(q+g+l-v+4, l-v+\frac{1}{2} ; q+g+\frac{9}{2} ; \frac{\frac{2 m}{\bar{\gamma}_{2}}+\frac{K+1}{\bar{\gamma}_{1}}-2 \sqrt{\frac{2 m(K+1)}{\bar{\gamma}_{2} \gamma_{1}}}}{\frac{K+1}{\bar{\gamma}_{2}}+\frac{2 m}{\frac{2 m(K+1)}{\gamma_{2} \gamma_{1}}}}\right)\right),
\end{aligned}
$$

respectively. Thus, by substituting (22) and (23) in (21), we obtained the second-order approximated ergodic capacity for ANC-based two-way AF relaying system in a mixed Rician and Nakagami- $m$ fading channels Figure 8.

\section{Asymptotic analysis}

In this section, we derive the asymptotic expression for the outage probability, which provides the insight view of the system performance and the diversity order. To obtain the asymptotic results for high SNR, we change some variables as, $z_{1}=\frac{\gamma_{\text {th }}}{\gamma_{1}}$ and $z_{2}=\frac{\gamma_{\text {th }}}{\gamma_{2}}$. For high SNR analysis, the CDF of $\gamma_{s_{1}}$ can be expressed as given in [13]

$$
F_{\gamma_{s I}}(z)=1-\left[1-F_{\gamma_{1}}\left(z_{1}\right)\right]\left[1-F_{\gamma_{2}}\left(z_{2}\right)\right]
$$

we can rewrite the CDF given in (5) for higher SNR analysis, by using the identity [10, Equation 8.354 .2 ]

$$
\begin{aligned}
\Gamma(a, x) & =\Gamma(a)-\sum_{n=0}^{\infty} \frac{(-1)^{n} x^{a+n}}{n !(a+n)} \\
& =\Gamma(a)-x^{a} \sum_{n=0}^{\infty} \frac{(-x)^{n}}{\Gamma(n+1)(a+n)}
\end{aligned}
$$

After applying the asymptotic expansion identity given in [14, Equation 14], (25) becomes

$$
\Gamma(a, x)=\Gamma(a)-\frac{x^{a}}{a}
$$

Using the result of (26) in (5), we get the CDF $F_{\gamma_{2}}\left(z_{2}\right)$ for high SNR as

$$
\begin{aligned}
F_{\gamma_{2}}\left(z_{2}\right) & =1-\left[\frac{\Gamma(m)-m^{m} z_{2}^{m} / m}{\Gamma(m)}\right] \\
& =\frac{m^{m} z_{2}^{m}}{m \Gamma(m)}+o\left(z_{2}^{m}\right)
\end{aligned}
$$

Also the CDF $F_{\gamma_{1}}\left(z_{1}\right)$ can be expressed by following the same procedure as in [15, Equation 15].

$$
F_{\gamma_{1}}\left(z_{1}\right)=e^{-K}(1+K) z_{1}+o\left(z_{1}\right)
$$

Hence, after some mathematical simplification and using (27), (28) in (24), we can express the outage probability of $\gamma_{s_{1}}$ at the high SNR as,

$$
F_{\gamma_{S_{1}}}(z)=\left\{\begin{array}{rr}
\frac{m^{m} z_{2}^{m}}{m \Gamma(m)}+e^{-K}(1+K) z_{1}+o\left(z_{1}\right) & m=1 \\
\frac{(m+1)^{m} z_{2}^{m}}{m \Gamma(m)}+e^{-K}(1+K) z_{1}+o\left(z_{2}^{m}\right) & m>1
\end{array}\right\},
$$

where $o(x)=\lim _{x \rightarrow 0} \frac{f(x)}{x}$ is defined in [11]. It is clear from (29) that the diversity order of the system is $\min [m, K]$. The diversity order will be verified in the Section 6.

\section{Simulation results and discussion}

In this section, we analyze the performance of the analog network coded two-way relay cooperative network by plotting the analytical curves along with the simulation results and comparing them over mixed Rician and Nakagami- $m$ fading channels. The performance of the system is analyzed by plotting the curves in terms of outage probability, average SER, ergodic capacity, and 
asymptotic outage probability for high SNR of the transmitted signal. We use Mathematica 7 to validate our close form analytical expressions and we build Mont-Carlo simulation using MATLAB for the system shown in Figure 1.

Figures 2, 3 and 4 show the outage probability performance of an ANC-based two-way relay network with different combinations of Nakagami- $m$ factor values $(1,2$, and 3), Rician $K$ factor values ( 0 , and $5 \mathrm{~dB}$ ), and threshold SNR $\gamma_{\mathrm{t} h}$ values $(0,5$, and $10 \mathrm{~dB})$. It can be observed from the figures that the increase in threshold SNR $\gamma_{\mathrm{t} h}$ degrades the performance of the system. For increasing values of $K$ and $m$ the performance of the system gets improved. The analytical and simulation results are in close proximity and validate the accuracy of analytical closed form expression. In high SNR region the asymptotic curves coincide with the analytical curves.

Figures 5, 6 and 7 present the average SER analysis over the mixed Rician and Nakagami- $m$ fading channels for BPSK and QPSK modulation schemes with different Nakagami- $m$ factor values $(1,2$, and 3 , respectively). The parameters for the modulation scheme are $a=1, b=1$ for BPSK and $a=1, b=0.5$ for QPSK. Analytical results are obtained by substituting these parameters in (16). The results show that the performance improves with the increasing values of Rician $K$ factor and Nakagami fading parameter $m$. From the examination of the slopes of SER curves, it is seen that, if we fix some value of $m$ at the MS and change the value of $K$ at the BS, then we can achieve a significant performance gain. In Figure 5 at average SER of $10^{-2}$, the system with $m=1, K=0 \mathrm{~dB}$ provides a performance gain of $1 \mathrm{~dB}$ over the system with $m=1$, $K=5 \mathrm{~dB}$ for BPSK constellation. Furthermore, the system with $m=1, K=10 \mathrm{~dB}$ is generating the same performance gain over the system with $m=1, K=5 \mathrm{~dB}$ at the average SER of $10^{-3}$. It can be shown that the diversity of system depends upon the channel conditions between the link $S_{1}$ and $R$. Here, we have improved the quality of the link between $S_{1}$ and $R$ by increasing the value of Nakagami fading parameter $m$ from 1 to 2 as shown in Figure 6. It is observed that the system is now exhibiting a larger performance gain at high SNR regions for different values of Rician factor $K$. For example, when average SER $=10^{-3}$, a system with $m=2, K=0 \mathrm{~dB}$ provides a performance gain of $4 \mathrm{~dB}$ over the system with $m=2, K=5 \mathrm{~dB}$ and a performance gain of $2 \mathrm{~dB}$ is achieved between $m=3$, $K=5 \mathrm{~dB}$ and $m=3, K=10 \mathrm{~dB}$ for BPSK modulation scheme. The same is true for $m=3$ as shown in Figure 7, we achieved the performance gain of $5 \mathrm{~dB}$ between $m=2$, $K=0 \mathrm{~dB}$ and $m=2$ and $K=5 \mathrm{~dB}$ and a performance gain of $3 \mathrm{~dB}$ is achieved for the system with $m=3, K=5$ $\mathrm{dB}$ over the system with $m=3, K=10 \mathrm{~dB}$ for both BPSK and QPSK modulation schemes. Moreover, the analytical closed form average SER expression is verified with Monte Carlos simulations.
Figure 8 shows the ergodic capacity for the different $m$ and $K$ values. The correctness of the analytical closed form expression for ergodic capacity is validated with Monte Carlos simulations. It is observed that increasing the Rician $K$ factor or Nakagami parameter $m$, improves the capacity of the system.

\section{Conclusion}

We analyzed the performance of network coding-based two-way relay network over mixed Rician and Nakagami$m$ asymmetric fading channels. We derived the closedform expressions for the outage probability, average SER using the derived $\mathrm{CDF}$, and ergodic capacity using the derived PDF to predict the performance of the proposed system. The outage probability for different values of threshold SNR $\gamma_{\text {th }}$ over mixed Rician and Nakagami- $m$ fading channels was calculated, which gives a better performance with lower amount of fading (high $m$ and $K$ values). The average SER analysis was carried out for BPSK, and QPSK modulation schemes. It is clear from the simulation results that for higher values of $m$ and $K$, better performance is achieved. In addition to that, the asymptotic outage probability for high SNR has been obtained, which provides a better understanding of the diversity order of the system.

\section{Competing interests}

Both authors declare that they have no competing interests.

\section{Authors' information}

Muhammad Hasanain Chaudary received his M.Sc. (Communications and Networks) in Computer Science and M.Sc. in Telecommunications from Bahria University, Islamabad, Pakistan and Asian Institute of Technology, Thailand respectively. He is currently working towards the PhD degree at the School of Engineering and Technology, Asian Institute of Technology, Thailand. Mr. Chaudarys research interests are in the general area of Broadband wireless networks and Signal processing for communication systems, and in particular Optimization for communication and networks, performance analysis of wireless communications systems, OFDM/OFDMA, MIMO, Network Coding and Cooperative systems.

Nandana Rajatheva received the B.Sc. degree in Electronic and

Telecommunication Engineering (with first class honors) from the University of Moratuwa, Sri Lanka, and the M.Sc. and Ph.D. degrees from the University of Manitoba, Canada, in 1987, 1991, and 1995, respectively. He is an Associate Professor of Telecommunications in the School of Engineering and Technology, Asian Institute of Technology, Thailand. Currently he is a visiting Professor at the Centre for Wireless Communications, University of Oulu, Finland. He is an Editor for the International Journal of Vehicular Technology (Hindawi). His research interests include performance analysis and resource allocation for relay, Network Coding, Cognitive radio and hierarchical cellular systems.

\section{Acknowledgements}

The first author would like to thank the Higher Education Commission (HEC) of Pakistan and COMSATS Institute of Information Technology, Lahore, Pakistan for facilitating and funding this work.

Received: 10 December 2011 Accepted: 29 May 2012

Published: 2 July 2012

\section{References}

1. R Ahlswede, N Cai, S-Y Li, R Yeung, Network information flow. IEEE Trans. Inf. Theory. 46(4), 1204-1216 (2000) 
2. S Wang, Q Song, X Wang, A Jamalipour, Rate and power adaptation for analog network coding. IEEE Trans. Veh. Technol. 60(5), 2302-2313 (2011)

3. J Yang, P Fan, TQ Duong, $X$ Lei, Exact performance of two-way AF relaying in Nakagami-m fading envoirnment. IEEE Trans. Wirel. Commun. 10(3), 980-987 (2011)

4. H Guo, J Ge, H Ding, Symbol error probability of two-way amplify-and-forward relaying. IEEE Commun. Lett. 15(1), 22-24 (2011)

5. TQ Duong, L-N Hoang, VNQ Bao, On the performance of two-way amplify-and-forward relay networks. IEICE Trans. Commun. E92-B(12), 3957-3959 (2009)

6. PK Upadhyay, S Prakriya, Performance of two-way opportunistics relaying with analog network coding over Nakagami-m fading. IEEE Trans. Veh. Technol. 60(4), 1965-1971 (2011)

7. W Li, J Li, P Fan, Network coding for two-way relaying networks over Rayleigh fading channels. IEEE Trans. Veh. Technol. 59(9), 4476-4488 (2010)

8. AK Gurung, FS Al-Qahtani, ZM Hussain, H Alnuweiri, Performance analysis of amplify-forward relay in mixed Nakagami- $m$ and Rician fading channels. in 2010 International Conference on Advanced Technologies for Communications (Ho Chi Minh City, 2010), pp. 321-326

9. HA Suraweera, RHY Louie, Y Li, GK Karagiannidis, B Vucetic, Two hop amplify-and-forward transmission in mixed Rayleigh and Rician fading channels. IEEE Commun. Lett. 13(4), 227-229 (2009)

10. IS Gradshteyn, M Ryzhik, Tables of Integrals, Series and Products (Elsevier Inc., Amsterdam, 2007)

11. NS Ferdinand, N Rajatheva, Performance analysis of imperfect channel estimation in MIMO two hop fixed gain relay network with beamforming. IEEE Commun. Lett. 15(2), 208-210 (2011)

12. SChen, W Wang, $X$ Zhang, $X$ Zhang, M Peng, $Y$ Li, Ergodic and outage capacity analysis of amplify-and-forward MIMO relay with OSTBCs. in IEEE Wireless Communications and Networking Conference (WCNC) (Sydney, 2010), pp. 1-6

13. H Ding, J Ge, Z Jiang, Asymptotic performance analysis of amplify and forward with partial relay selection in rician fading. IET Electron. Lett. 46(3), 263-264 (2010)

14. C Zhong, K-K Wong, S Jin, M Alouini, T Ratnarajah, Asymptotic analysis for Nakagami-m fading channels with relay selection. in IEEE International Conference on Communications (ICC) (Kyoto, 2011), pp. 1-5

15. S Chen, F Liu, X Zhang, Y Han, D Yang, On the performance of two-hop amplify and forward relay networks with beamforming over Rayleigh-Rician fading channels. in IEEE Vehicular Technology Conference Fall (VTC 2010-Fall) (Ottawa, 2010), pp. 1-4

doi:10.1186/1687-1499-2012-209

Cite this article as: Chaudary and Rajatheva: Performance analysis of analog network coding based two-way amplify-and-forward system in mixed Rician and Nakagami- $m$ fading environment. EURASIP Journal on Wireless Communications and Networking 2012 2012:209.

\section{Submit your manuscript to a SpringerOpen ${ }^{\odot}$ journal and benefit from:}

- Convenient online submission

- Rigorous peer review

- Immediate publication on acceptance

- Open access: articles freely available online

- High visibility within the field

- Retaining the copyright to your article

Submit your next manuscript at springeropen.com 